\title{
Acknowledgement to scientific referees 2020
}

\author{
Pierre Lachapelle
}

Published online: 13 February 2021

(C) The Author(s), under exclusive licence to Springer-Verlag GmbH, DE part of Springer Nature 2021

As the Editors of Documenta Ophthalmologica, we would like to sincerely thank all the referees for the time and dedication given to reviewing manuscripts for Documenta Ophthalmologica in 2020.

The greatest responsibility of a scientific journal is to ensure that all contributions submitted for publication are rigorously but fairly reviewed. Therefore, we gratefully acknowledge the valuable support of our collaborators, and we would like to take this opportunity to share some positive results and new forthcoming challenges.

Documenta Ophthalmologica has been growing over the last few years:

Submissions significantly increased since 2017. However, turnaround times are very good (27 days from submission to first decision, 12 days from acceptance to online publication).

Yearly article downloads are constantly increasing. In 2020, we had more than 80,000 downloads.

The Impact Factor slightly decreased to 1.294 (2019).

We look forward to maintaining our collaborative efforts to ensure that the impact of Documenta Ophthalmologica continues to grow and our journal continues to attract manuscripts of exceptionally high

P. Lachapelle $(\square)$

Montreal, Canada

e-mail: pierre.lachapelle@mcgill.ca standards of research in the field of basic and clinical electrophysiology of vision.

With kind regards,

Mineo Kondo

Jan Kremers

Daphne L. McCulloch

Suresh Viswanathan

(Associate Editors)

Pierre Lachapelle

(Editor-in-Chief)

We are indebted to the following experts who reviewed papers for the journal in 2020. Their expertise and invaluable assistance contributed to maintain a high-scientific standard for the Journal. Reviewers are listed in alphabetical order.

James Akula, UNITED STATES

Khaldoon Al-Nosairy, GERMANY

Lucia Ambrosio, UNITED STATES

Rola Ba-Abbad, UNITED KINGDOM

Michael Bach, GERMANY

So Hyun Bae, KOREA, REPUBLIC OF

Sherry Bass, UNITED STATES

David Birch, UNITED STATES

Mei Ying Boon, AUSTRALIA

Jelka Brecelj, SLOVENIA

Mitchell Brigell, UNITED STATES

Scott Brodie, UNITED STATES

Giuseppe Casalino, ITALY 
Henry Chan, CHINA

Enid Chelva, AUSTRALIA

Kyung Choi, KOREA, REPUBLIC OF

Paul Constable, AUSTRALIA

Allison Dorfman, CANADA

Arlene Drack, UNITED STATES

Benedetto Falsini, ITALY

Beatrix Feigl, AUSTRALIA

Anthony Fisher, UNITED KINGDOM

Laura Frishman, UNITED STATES

Mercedes Gauthier, CANADA

Mathieu Gauvin, CANADA

Melanie Gillingham, UNITED STATES

Vivienne Greenstein, UNITED STATES

John Grigg, AUSTRALIA

Ruth Hamilton, UNITED KINGDOM

Wendy Harrison, UNITED STATES

Takaaki Hayashi, JAPAN

Sven Heinrich, GERMANY

John Hetling, UNITED STATES

Michael Hoffmann, GERMANY

Graham Holder, SINGAPORE

Karen Holopigian, UNITED STATES

Folkert Horn, GERMANY

Cord Huchzermeyer, GERMANY

Brett Jeffrey, UNITED STATES

Mary Johnson, UNITED STATES

Makita Jun, JAPAN

Shuhei Kameya, JAPAN

David Keating, UNITED KINGDOM

Kamron Khan, UNITED KINGDOM

Naheed Khan, UNITED STATES

Safal Khanal, NEW ZEALAND

Jin Young Kim, KOREA, REPUBLIC OF

Ungsoo Kim, KOREA, REPUBLIC OF

Alexander Klistorner, AUSTRALIA

Rob Koenekoop, CANADA

Hiroyuki Kondo, JAPAN

Mineo Kondo, JAPAN

Jan Kremláček, CZECH REPUBLIC

Anne Kurtenbach, GERMANY

Pierre Lachapelle, CANADA

Bo Lei, CHINA

Shiying Li, CHINA

Wu Liu, CHINA

Wojciech Lubinski, POLAND

Shigeki Machida, JAPAN

Ted Maddess, AUSTRALIA

Omar Mahroo, UNITED KINGDOM
Michael Marmor, UNITED STATES

J. McAnany, UNITED STATES

Daphne McCulloch, CANADA

Declan McKeefry, UNITED KINGDOM

Gen Miura, JAPAN

Atsushi Mizota, JAPAN

Kiyofumi Mochizuki, JAPAN

Freya Mowat, UNITED STATES

Balazs Nagy, HUNGARY

Stephen Neuhauss, SWITZERLAND

James Odom, UNITED STATES

Anja Palmowski-Wolfe, SWITZERLAND

Athanasios Panorgias, UNITED STATES

Galina Paramei, UNITED KINGDOM

Neil Parry, UNITED KINGDOM

Neal Peachey, UNITED STATES

Ido Perlman, ISRAEL

Anna Polosa, CANADA

Vittorio Porciatti, UNITED STATES

Julie Racine, UNITED STATES

Aparna Raghuram, UNITED STATES

William Ridder III, UNITED STATES

Anthony Robson, UNITED KINGDOM

Idaira Sanchez-Santos, MEXICO

Marc Sarossy, AUSTRALIA

Jongmo Seo, KOREA, REPUBLIC OF

Jerome Sherman, UNITED STATES

Kei Shinoda, JAPAN

Benjamin Smith, CANADA

Richard Smith, UNITED KINGDOM

Katarina Stingl, GERMANY

Torsten Straßer, GERMANY

Ruifang Sui, CHINA

Maja Sustar, SLOVENIA

Atsuhiro Tanikawa, JAPAN

Manca Tekavcic-Pompe, SLOVENIA

Dorothy Thompson, UNITED KINGDOM

Alma Tormene, ITALY

Francois Tremblay, CANADA

Christopher Tyler, UNITED KINGDOM

Radouil Tzekov, UNITED STATES

Shinji Ueno, JAPAN

Maria van Genderen, NETHERLANDS

James Ver Hoeve, UNITED STATES

Ajoy Vincent, CANADA

Algis Vingrys, AUSTRALIA

Suresh Viswanathan, UNITED STATES

Avery Weiss, UNITED STATES

Tom Wright, CANADA 
John Wroblewski, UNITED STATES

Shuichi Yamamoto, JAPAN

Nilufer Yesilirmak, TURKEY

Phillip Yuhas, UNITED STATES
Publisher's Note Springer Nature remains neutral with regard to jurisdictional claims in published maps and institutional affiliations. 